\title{
Real-time Polymerase Chain Reaction System Using a Camera for Open Platform
}

\author{
Ji-Soo Hwang, ${ }^{1,2}$ Chan-Young Park, ${ }^{1,2}$ Hye-Jeong Song, ${ }^{1,2}$ \\ Yu-Seop Kim, ${ }^{1,2}$ Jongwon Kim, ${ }^{3}$ and Jong-Dae Kim ${ }^{1,2^{*}}$ \\ ${ }^{1}$ Department of Convergence Software, Hallym University, \\ 1 Hallymdaehak-gil, Chuncheon-si, Gangwon-do 24252, Korea \\ ${ }^{2}$ Bio-IT Research Center, Hallym University, 1 Hallymdaehak-gil, Chuncheon-si, Gangwon-do 24252, Korea \\ ${ }^{3}$ Daegu-Gyeongbuk Medical Innovation Foundation, 88 Dongnae-ro, Dong-gu, Daegu City 41061, Korea
}

(Received April 16, 2018; accepted March 20, 2019)

Keywords: fluorescence detection, USB camera, real-time polymerase chain reaction, open platform

In real-time polymerase chain reaction (RT-PCR), the result of the amplification of DNA can be seen through changes in fluorescence brightness sensed during the PCR cycles. To detect the changes in fluorescence intensities, commercial real-time PCR thermocyclers mainly use photodiodes for detection. However, our previous study showed that a digital single lens reflex (DSLR) camera instead of a photodiode can detect changes in fluorescence intensity as well as differentiate cases of unsatisfactory experimental results. Although the fluorescence detection was successful, the adoption of the DSLR camera was problematic in terms of size and cost. In this paper, we show that fluorescence detection is also possible using a small USB camera for open platforms instead of bulky DSLR cameras.

\section{Introduction}

Real-time polymerase chain reaction (RT-PCR) is used to determine whether amplification is possible by real-time quantitative analysis using fluorescence detection during the amplification of nucleic acids such as DNA or RNA. ${ }^{(1-3)}$ Commercial RT-PCR instruments used in large laboratories or hospitals now use bulky thermocyclers because they amplify DNA or RNA using a multiwell plate. ${ }^{(4)}$ In addition, these devices typically use large filter wheels and expensive, high-performance CCD cameras for multichannel fluorescence detection. Even equipment that uses relatively inexpensive photodiodes often consists of a fluorescence detection system that has complex optical structures that include a variety of optical elements such as objective lenses, eyepieces, dichroic mirrors, and pinholes. ${ }^{(5-7)}$

Although a case of using the camera of a smartphone has been reported as a sensor for detecting fluorescence, in most cases, a high-resolution camera including a CCD camera is used and a complicated optical component is used as described above. ${ }^{(5)}$ Owing to the optical components of the detection device, they tend to be large and expensive. Therefore, a lot of research has been carried out to manufacture such a device in a miniaturized and low-cost manner. ${ }^{(8,9)}$

*Corresponding author: e-mail: kimjd@hallym.ac.kr https://doi.org/10.18494/SAM.2019.2285 
Research and development is underway to amplify gene samples and create microfluidic channels on the chip to detect fluorescence. The disposable PCR chips reported in previous studies were made by attaching a plastic housing to easily inject reagents into microfluidic channels made by attaching packing tape, black double-sided tape, and a plastic cover to a printed circuit board (PCB). ${ }^{(10-21)}$

In general, photodiodes are used for the fluorescence detection of PCR chips; nevertheless, in previous studies, cameras were used instead of photodiodes to observe the state of the reaction chamber as well as the degree of DNA amplification. ${ }^{(1)}$ For example, we found that local boiling was one of the causes of amplification failure for our PCR chips, which could not be identified without the camera detection.

Our previous study used a digital single lens reflex (DSLR) camera to construct a fluorescence detection system for PCR chips and showed the results of real-time fluorescence detection of Chlamydia trachomatis (CT) DNA. The DSLR camera used as a detection device has an advantage that a high-quality image can be obtained, but the size of the camera module is considerably large because the camera is expensive and uses a bulky lens, an extension tube, and a camera body. Therefore, it is not suitable as a fluorescence detection device of point-ofcare devices aimed at most PCR chips. ${ }^{(11)}$

In this work, a real-time fluorescence detection system was constructed by replacing DSLR cameras with open-platform-based smartphone cameras. Since the development of the smartphone, the ultrahigh-resolution and ultrasmall camera module can be easily obtained at a low price, so that the proposed system can be constituted by one-fifth of the size of the existing system. The proposed RT-PCR system showed the fluorescence detection performance comparable to commercial RT-PCR instruments.

\section{Materials and Methods}

\subsection{RT-PCR system}

Figure 1 shows the RT-PCR system proposed in this paper. The system consists of a local system part that mainly controls the thermal cycling of the PCR chip and a host PC part that processes the user interface. For fluorescence detection, the excitation LED is controlled by the local system, while the camera is connected directly to the host. The blue LED and the camera module constitute a fluorescence detection part together with two types of filters: one is for the excitation and the other is for the emission. The local system can be controlled from the PC via the USB interface. ${ }^{(12,22-24)}$

As shown in Fig. 2(a), the emission filter (interference filter, Edmund Optics) was installed in front of a small smartphone camera module (Sony IMX179 mini surveillance camera) and the excitation filter (interference filter, Edmund Optics) was fixed in front of the 9600 mcd blue LED. Figure 2(b) shows a block diagram of the optical structure of the proposed fluorescence detector system. The reaction chamber was illuminated diagonally using a blue LED. Even though a diagonal illumination method is a relatively simple technique to implement, it has a problem that the reaction chamber is not uniformly illuminated. However, it does not seem 


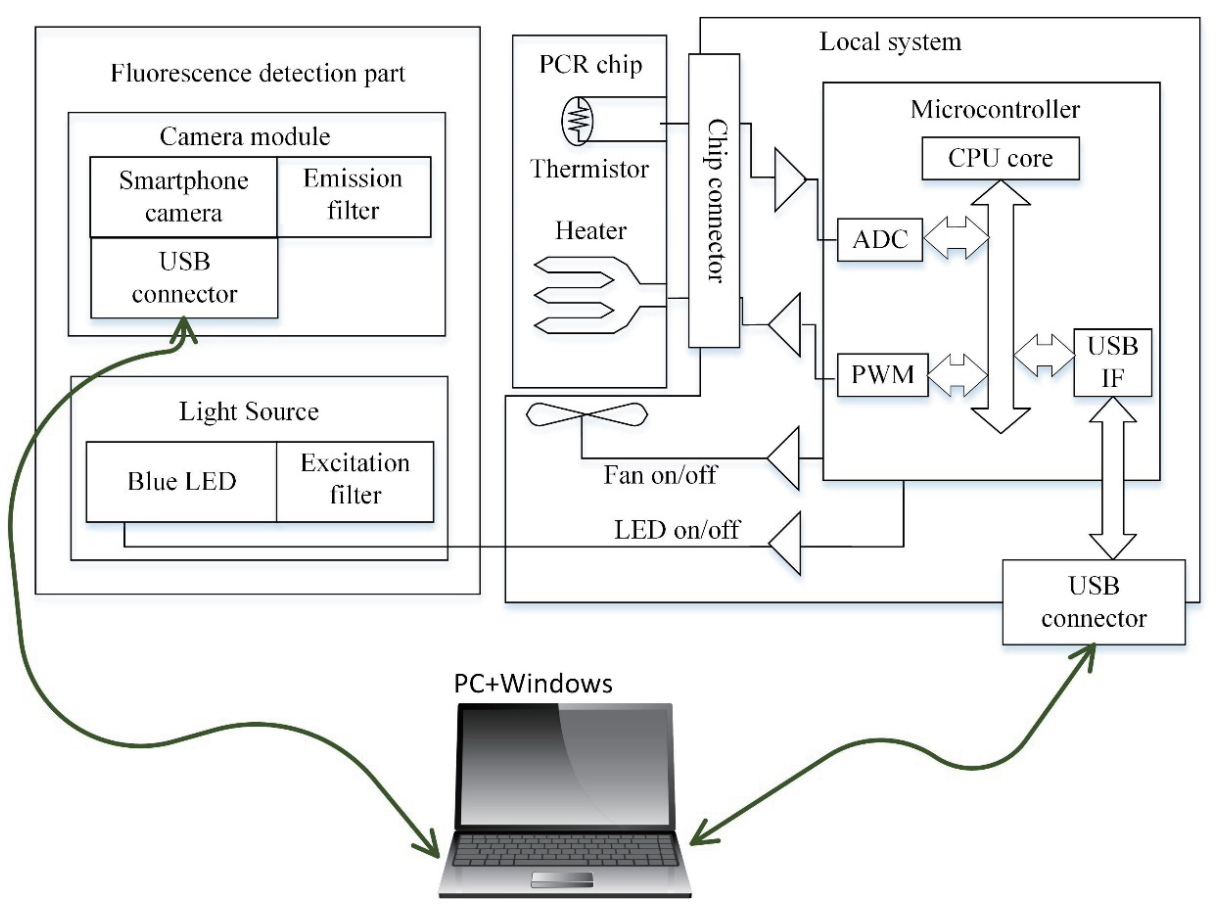

Fig. 1. (Color online) Proposed RT-PCR system.

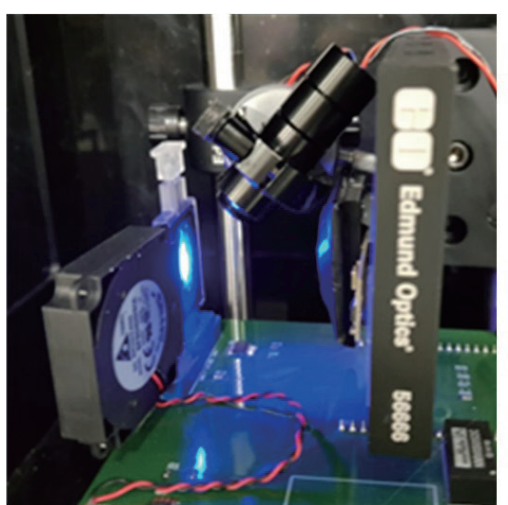

(a)

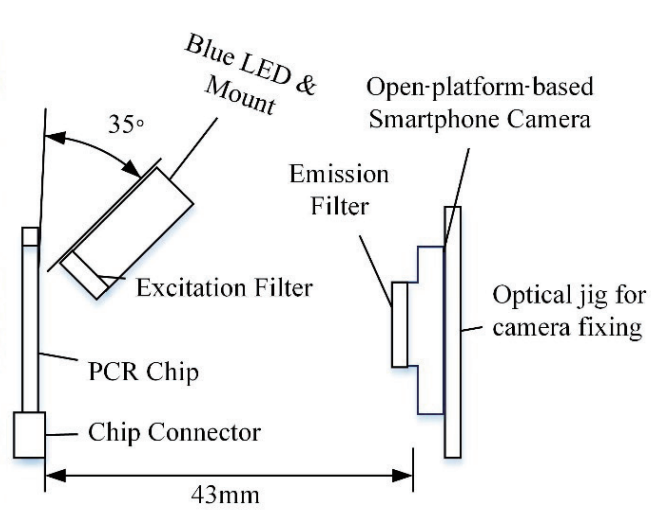

(b)

Fig. 2. (Color online) (a) Optical setup for the proposed RT-PCR system and (b) its block diagram.

to be a problem because we calculated the average brightness of the reaction chamber area to measure fluorescence quantities in this paper.

Since the minimum working distance for most DSLR cameras is large, the distance from the camera body to the chip was about $240 \mathrm{~mm}$ in the previous study. However, owing to the short working distance of the smartphone cameras, the proposed system could reduce the distance to about $50 \mathrm{~mm}$. 


\subsection{PCR chip structure}

Figure 3 shows the structure of the PCR chip used in this study. Figure 3(a) shows the assembly diagram of the chip, and Fig. 3(b) shows the cross section. The reaction chamber was made by stacking a $50-\mu \mathrm{m}$-thick packing tape, a $400-\mu \mathrm{m}$-thick black double-sided tape, and a $200-\mu \mathrm{m}$-thick polycarbonate film on a matte black PCB substrate.

To facilitate reagent injection, a plastic housing with an inlet and an outlet hole was attached to the chip with a transparent double-sided tape of $200 \mu \mathrm{m}$ thickness resulting in a six-layered chip.

The middle portion of the PCB substrate has a heating pattern capable of heating the reaction chamber. Adopting black matte ink as the solder mask and white silk legend coating over the heater pattern maximizes the reflection of the emitted light and minimizes unwanted reflections. ${ }^{(10,11,23)}$

\subsection{Experimental method}

Table 1 shows the reagent composition used in the experiment. $5.8 \mu \mathrm{L}$ of CT DNA at a concentration of $1 \mathrm{ng} / \mu \mathrm{L}, 10.8 \mu \mathrm{L}$ of a master mix (TaqMan Gene Expression, ABI), $10.8 \mu \mathrm{L}$ of a primer mix with a concentration of $10 \mathrm{pmole} / \mu \mathrm{L}$ consisting of primers $\mathrm{F}, \mathrm{R}$, and probe, and $9 \mu \mathrm{L}$ of distilled water were mixed and injected into the PCR chip.

RT-PCR was performed using the two-step protocol shown in Table 2 of the experimental setup, as shown in Fig. 2(a). In this paper, the LED was turned on two seconds before the

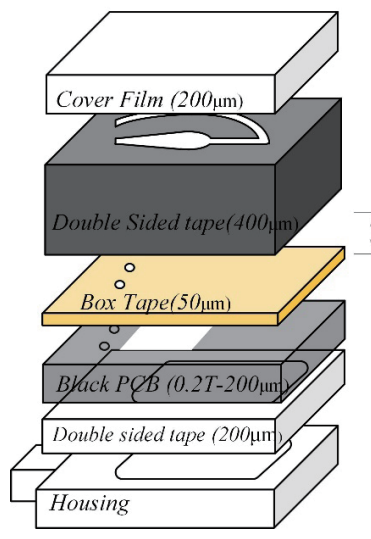

(a)

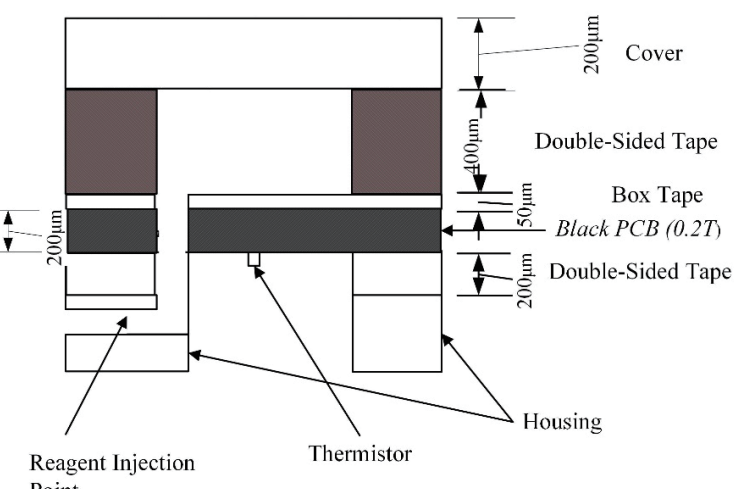

(b)

Fig. 3. (Color online) (a) PCR chip structure assembly diagram and (b) its cross section.

Table 1

Reagent creation for RT-PCR.

\begin{tabular}{lcr}
\hline Reagents & Concentration & Volume \\
\hline CT DNA & $1 \mathrm{ng} / \mu \mathrm{L}$ & $5.4 \mu \mathrm{L}$ \\
Master mix & & $10.8 \mu \mathrm{L}$ \\
Primer mix & 10 pmole $/ \mu \mathrm{L}$ & $10.8 \mu \mathrm{L}$ \\
Distilled water & & $9 \mu \mathrm{L}$ \\
\hline Total volume & & $36 \mu \mathrm{L}$ \\
\hline
\end{tabular}

Table 2

PCR protocol used in the RT-PCR test.

\begin{tabular}{lccc}
\hline PCR Step & Temperature & Time & No. of cycles \\
\hline Preincubation & $50{ }^{\circ} \mathrm{C}$ & $2 \mathrm{~min}$ & \multirow{2}{*}{1} \\
Preheating & $95{ }^{\circ} \mathrm{C}$ & $10 \mathrm{~min}$ & \\
\hline Denaturation & $95{ }^{\circ} \mathrm{C}$ & $15 \mathrm{~s}$ & 40 \\
Annealing & $60{ }^{\circ} \mathrm{C}$ & $1 \mathrm{~min}$ & \\
\hline
\end{tabular}


completion of the $60{ }^{\circ} \mathrm{C}$ annealing step, and the LED was turned off when the annealing step was finished. The 40 images for the entire cycles of the RT-PCR protocol were analyzed by calculating the mean fluorescence intensity in the reaction chamber area using the Matlab program.

To evaluate the proposed system, the prepared reagents were tested using Roche's LightCycler ${ }^{\circledR} 480$ instrument. To verify the prepared reagent and obtain reference curves of the fluorescence brightness change according to reagent concentration, the 10- and 100-fold dilutions of the prepared reagent were tested together.

\section{Experimental Results}

Figure 4 is a fluorescence detection image using the proposed RT-PCR system. In the images taken in the early cycle, they seem to be slightly darkened, but maintain a similar brightness up to about 10 cycles, then the brightness increases abruptly from 13 to 15 cycles, and the change in brightness is noticeably different in the subsequent cycles. Comparison of the brightness of the first and last cycles visually confirms that there is considerable change. Even
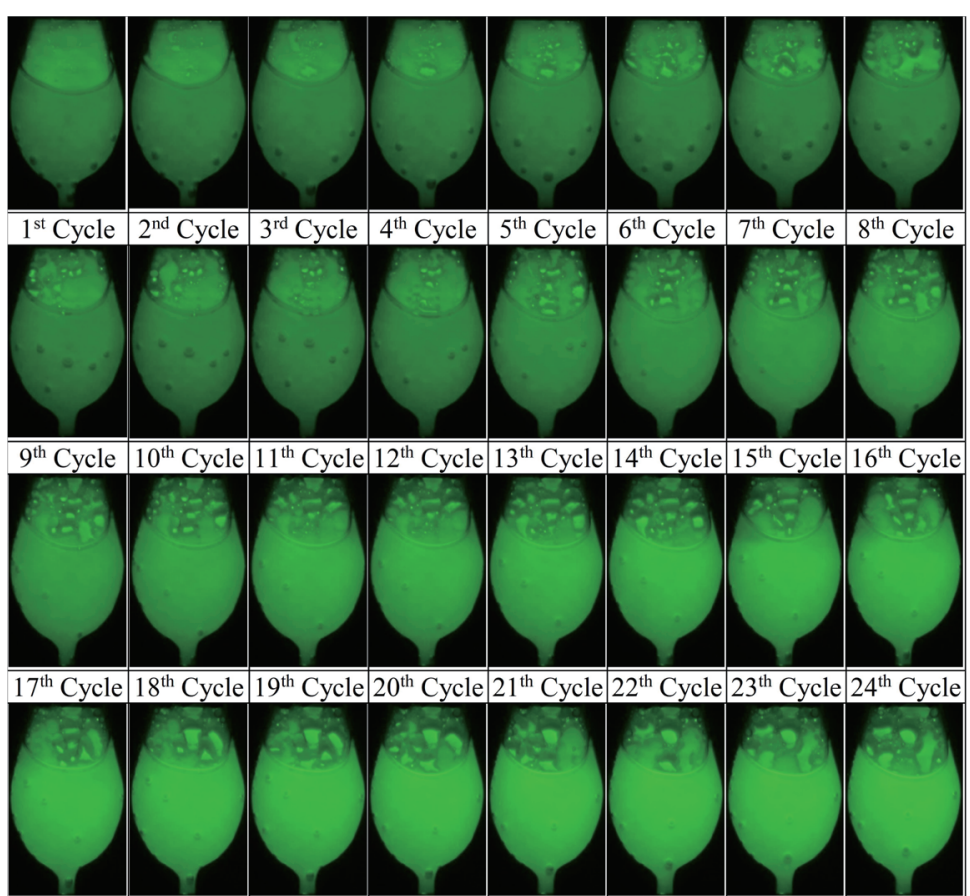

$25^{\text {th }}$ Cycle $26^{\text {th }}$ Cycle $27^{\text {th }}$ Cycle $28^{\text {th }}$ Cycle $29^{\text {th }}$ Cycle $30^{\text {th }}$ Cycle $31^{\text {th }}$ Cycle $32^{\text {th }}$ Cycle

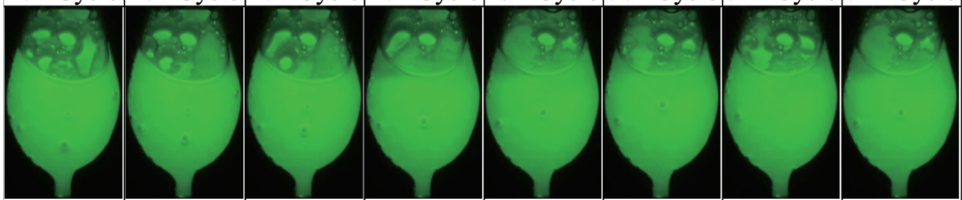

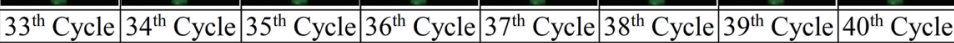

Fig. 4. (Color online) Captured images during RT-PCR. 
though there is a large air bubble on the upper part and several small bubbles in the lower part of the reaction chamber, there is no considerable effect on the average brightness as shown in Fig. 5.

Figure 5 shows the average brightness of the images in Fig. 4. The result shows that the average fluorescence intensity starts to increase rapidly in around the 12th to 13th cycles.

Figure 6 shows the results of RT-PCR with LightCycler ${ }^{\circledR} 480$. The rapid increase in fluorescence curves for 10- and 100-fold dilutions was observed approximately 3.3 and 6.6

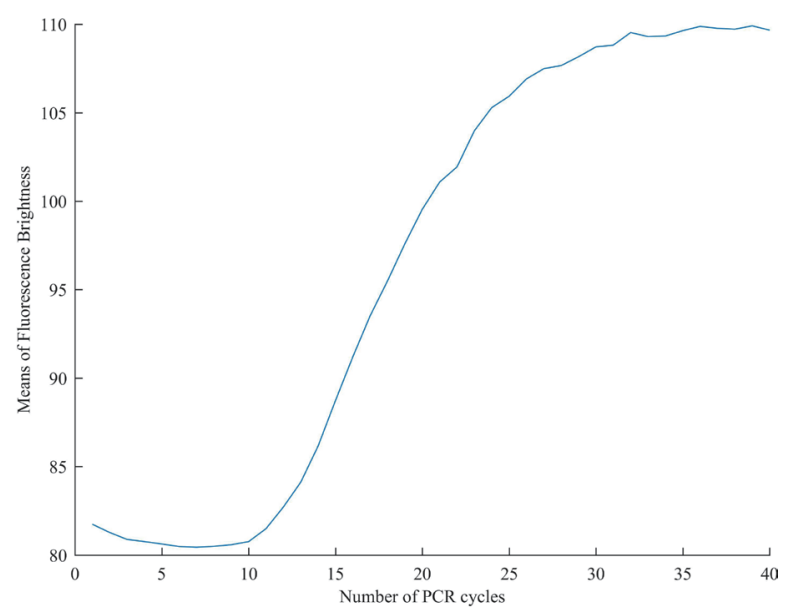

Fig. 5. (Color online) Fluorescence brightness analysis of CT DNA (1 ng/ $\mu \mathrm{L})$.

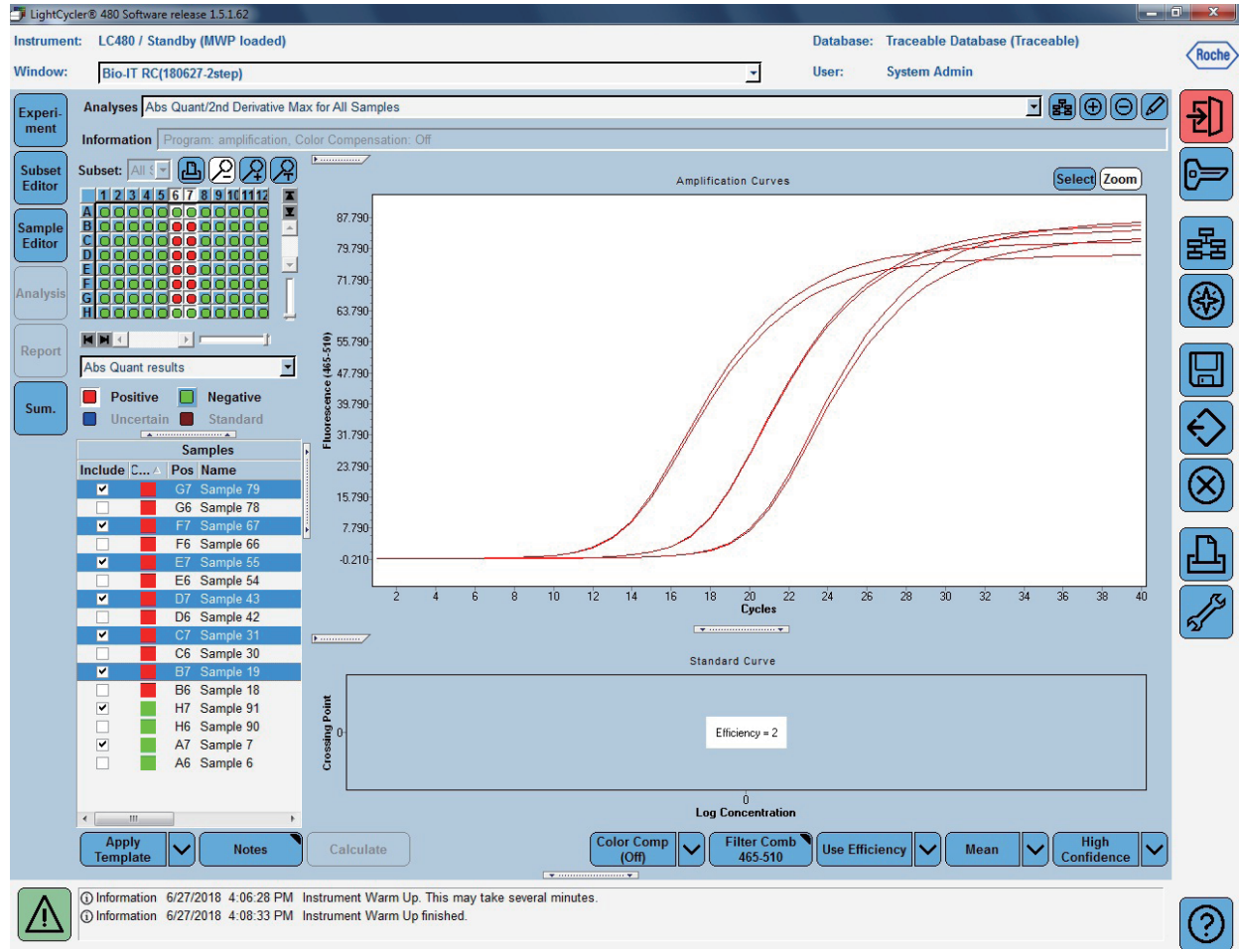

Fig. 6. (Color online) RT-PCR analysis report of CT DNA $\left(1,10^{-1}\right.$, and $\left.10^{-2} \mathrm{ng} / \mu \mathrm{L}\right)$ using LightCycler ${ }^{\circledR} 480$ (Roche). 
cycles later than with the original $1 \mathrm{ng} / \mu \mathrm{L}$ reagent, showing that there is no problem with the prepared reagent. In addition, it could be seen that the curve for the $1 \mathrm{ng} / \mu \mathrm{L}$ concentration increased rapidly at $12-13$ cycles similar to the curve shown in Fig 5. These results implied that the proposed system can be used as a real-time PCR device.

\section{Conclusions}

RT-PCR systems that are currently in commercial use are generally designed to perform fluorescence detection tests using expensive CCD cameras or photodiodes. Photodiodes are very small in size and cost less than expensive CCD cameras, making system construction costeffective. However, when constructing a system using a photodiode, it is common to construct a system with a complex optical structure using various optical components such as an objective lens, an eyepiece, a pinhole, and a dichroic mirror. Furthermore, as the optical components require certain distances from each other, it is difficult to make the system compact. Moreover, it is difficult to find and solve the errors or problems in the experiment because the photodiodes can only measure the average brightness.

In a previous study, we constructed an RT-PCR system using a DSLR camera as a fluorescence detection sensor and showed that it is possible to monitor reagent chambers as well as fluorescence detection. However, the DSLR camera is disadvantageous in terms of price compared with the photodiode, and the volume of the entire camera is bulky because the lens and the extension tube for the macro lens should be combined with the camera body. Therefore, it is not easy to manufacture a small system and not proper for point-of-care systems.

In this paper, we conducted a fluorescence detection test using a small, high-resolution smartphone camera instead of a DSLR camera. With the recent development of smart phones, miniaturization and high-resolution cameras are becoming popular, so small RT-PCR systems using these products can be implemented easily and inexpensively. We were also able to produce one-fifth smaller systems than in previous studies.

To determine the validity of the fluorescence detection, a comparison experiment was performed using a commercial RT-PCR system. We found that the fluorescence intensity of the proposed system began to increase at the cycle similar to that of the commercial one. However, further research is required in terms of analysis, such as obtaining threshold cycles and crossing points for a more accurate quantitative comparison.

\section{Acknowledgments}

This research was supported by Hallym University Research Fund, 2018(HRF-201806-012).

\section{References}

1 S. A. Bustin: Encyclopedia of Diagnostic Genomics and Proteomics, J. Fuchs and M. Podda, Eds. (Marcel Dekker Inc, New York, 2005) p. 1117.

2 S. Bustin, V. Benes, T. Nolan, and M. Pfaffl: J Mol. Endocrinol. 34 (2005) 597.

3 C. A. Heid, J. Stevens, K. J. Livak, and P. M. Williams: Genome Res. 6 (1996) 986. 
4 C. Y. Park, M. S. Lee, Y. S. Kim, H. J. Song, and J. D. Kim: Int. J. Eng. Technol. Innovation 8 (2018) 191.

5 H. Zhu, S. O. Isikman, O. Mudanyali, A. Greenbaum, and A. Ozcan: Lab Chip 13 (2013) 51.

6 L. Golonka, H. Roguszczak, T. Zawada, J. Radojewski, I. Grabowska, M. Chudy, A. Dybko, Z. Brzozka, and D. Stadnik: Sens. Actuators, B 111 (2005) 396.

7 N. M. M. Pires, T. Dong, U. Hanke, and N. Hoivik: Sensors 14 (2014) 15458.

8 Q. Xiang, B. Xu, R. Fu, and D. Li: Biomed. Microdevices 7 (2005) 273.

9 L. K. Wang, J.-Y. Shieh, and K. Huang: Int. J. Eng. Technol. Innovation 2 (2012) 163.

10 J. S. Hwang, S.-. Kim, Y. S. Kim, H. J. Song, C. Y. Park, and J. D. Kim: Int. J. Control Autom. 8 (2015) 117.

11 J. S. Hwang, Y. S. Kim, H. J. Song, J. D. Kim, and C. Y. Park: Technol. Health Care 24 (2016) S139.

12 J. S. Hwang, J. D. Kim, Y. S. Kim, H. J. Song, and C. Y. Park: Sens. Mater. 30 (2018) 397.

13 M. Focke, D. Kosse, C. Müller, H. Reinecke, R. Zengerle, and F. von Stetten: Lab Chip 10 (2010) 1365.

14 D. Patko, Z. Mártonfalvi, B. Kovacs, F. Vonderviszt, M. Kellermayer, and R. Horvath: Sens. Actuators, B 196 (2014) 352.

15 Y. S. Shin, K. Cho, S. H. Lim, S. Chung, S.-J. Park, C. Chung, D.-C. Han, and J. K. Chang: J. Micromech. Microeng. 13 (2003) 768.

16 P. Neuzil, J. Pipper, and T. M. Hsieh: Mol. Biosyst. 2 (2006) 292.

17 K. Sun, A. Yamaguchi, Y. Ishida, S. Matsuo, and H. Misawa: Sens. Actuators, B 84 (2002) 283.

18 Z. Q. Zou, X. Chen, Q. H. Jin, M. S. Yang, and J. L. Zhao: J. Micromech. Microeng. 15 (2005) 1476.

19 A. M. Foudeh, T. F. Didar, T. Veres, and M. Tabrizian: Lab Chip 12 (2012) 3249.

20 D. Moschou, N. Vourdas, G. Kokkoris, G. Papadakis, J. Parthenios, S. Chatzandroulis, and A. Tserepi: Sens. Actuators, B 199 (2014) 470.

21 I. Schneegaß, R. Bräutigam, and J. M. Köhler: Lab Chip 1 (2001) 42.

22 J. Kim, J.-D. Kim, and G. Lee: SPIE MOEMS-MEMS 7929 (2011) 79290 Y.

23 C.-Y. Park, J.-D. Kim, J.-H. Ku, Y.-S. Kim, H.-J. Song, and J. Kim: Sens. Lett. 10 (2012) 1197.

24 C. E. Wills: Electro/94 Int. Conf. Proc. Combined Volumes (1994) 415.

\section{About the Authors}

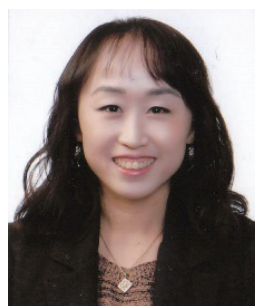

Ji-Soo Hwang received her M.S. and Ph.D. degrees in Computer Engineering from Hallym University. She is currently a research professor in Bio-IT Research Center at Hallym University. Her recent research is focused on biomedical systems and bioinformatics.

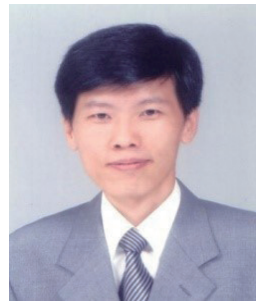

Chan-Young Park received his B.S. degree from Seoul National University and his M.S. and Ph.D. degrees from Korea Advanced Institute of Science and Technology in 1989 and 1995, respectively. From 1991 to 1999, he worked at Samsung Electronics. He is currently a professor in the School of Software at Hallym University. His research interests are in the areas of Bio-IT convergence, intelligent transportation systems, and sensors.

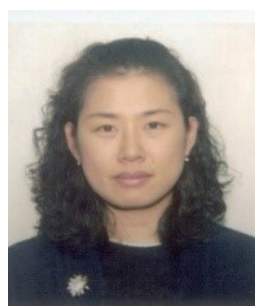

Hye-Jeong Song received her Ph.D. degree in Computer Engineering from Hallym University. She is currently a research professor in the Bio-IT Research Center at Hallym University. Her recent research is in the areas of biomedical systems and bioinformatics. 


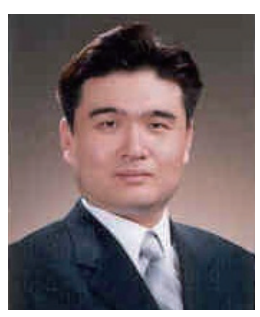

Yu-Seop Kim received his Ph.D. degree in Computer Engineering from Seoul National University. He is currently a professor in the School of Software at Hallym University. His research interests are in the areas of bioinformatics, computational intelligence, and natural language processing.

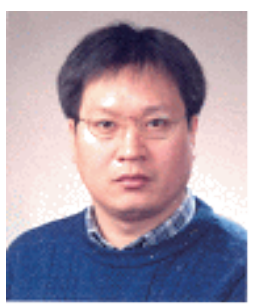

Jongwon Kim received his B.S. and M.S. degrees in Physics from Seoul National University in 1985 and 1987, respectively. He received his Ph.D. degree in Biomedical Engineering from Seoul National University in 1992. Since 2018, he has been the director of the medical development center at Daegu Gyeongbuk Advanced Medical Industry Promotion Foundation. His research interests include biomedical systems and bioinformatics.

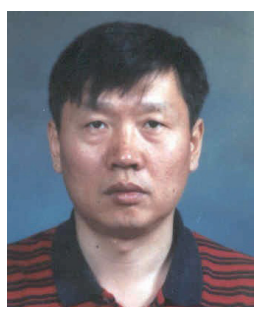

Jong-Dae Kim received his M.S. and Ph.D. degrees in Electrical and Electronics Engineering from Korea Advanced Institute of Science and Technology, Seoul, Korea, in 1984 and 1990, respectively. He worked for Samsung Electronics from 1988 to 2000 as an electronic engineer. $\mathrm{He}$ is currently a professor in the School of Software at Hallym University. His recent interests are focused on biomedical systems and bioinformatics. 